\title{
Conceptual Guideway Structural Design for MAGLEV High-Speed Ground Transportation System
}

\author{
Can Balkaya*+, W.J. Hall** \\ *Department of Civil Engineering, Faculty of Engineering and Architecture, Istanbul Gelisim University, Istanbul, Turkey \\ **Department of Civil and Environmental Engineering, University of Illinois at Urbana-Champaign, Urbana, IL, USA.
}

(cbalkaya@gelisim.edu.tr, wjefhall@comcast.net)

‡Corresponding Author; Can Balkaya, Department of Civil Engineering, Istanbul Gelisim University, Istanbul, Turkey

Tel: +90 212422 7020, Fax: +90 212422 7401, cbalkaya@ gelisim.edu.tr

Received: 27.04.2017 Accepted: 21.06.2017

\begin{abstract}
The conceptual guideway structural designs for MAGLEV (magnetic-levitation) high-speed ground transportation system are discussed by considering four different guideway designs of Bechtel, Magneplane, Grumman and Foster-Miller. The important aspects of the conceptual designs as well as some of the apparent shortcomings that will need attention in the design studies are emphasized. In this context the technical assessments and design observations may be considered for the guideway conceptual designs are given in this study.
\end{abstract}

Keywords Guideway, MAGLEV, Structural design, High-speed, Transportation system

\section{Introduction}

High-speed rails were mainly constructed on guideway structures with fast progress of train technology as MAGLEV (magnetic-levitation) for ground transportation system. The assessment studies have involved detailed review of four major conceptual designs namely those by Bechtel Corporation [2], Magneplane International Inc. [3], Grumman Aerospace Corporation [4], and Foster-Miller Inc. [5]. The assessment observations center on major issues addressed in the conceptual design reports. The evaluation has been prepared purposely to emphasize the positive aspects of the conceptual designs, as well as some of the apparent shortcomings that will need additional attention in design studies in the years ahead [6]. In this context the assessments may be considered to be somewhat general in nature at this point in time, for in almost all cases for the guideway conceptual designs presented, the technical details provided were, at most, of a 'general nature'.

With respect to the technical observations arising from these studies, authors offer the following observations. Four major conceptual design studies considered with concentration on subtopics in five major areas, namely (1) route; (2) environmental concerns; (3) design criteria and preliminary design; (4) vehicle-system interface; and (5) maintenance/repair.

For the most part, as would be expected and is appropriate, major attention has been devoted to the guideway, and various factors that affect the guideway design, including operation and maintenance of the guideway system, vary between the various studies, and admittedly are difficult to quantify until such time as specific route is selected. It is safe to say that this area of the system design studies, namely the guideway system, is going to require a great deal more effort in the years ahead as the approaches in US an operating maglev high-speed ground transportation system.

\section{Technical Assessment and Design Observations}

\subsection{Route}

All of the conceptual designers gave considerable attention to a hypothetical route in the sense of looking at system requirements generally, and then specifically 
alignment, curvature, tilt, and related factors pertaining to the routing and operation of the MAGLEV system, especially as it would relate to the performance of the vehicles.

With reference to 'best ride' assessment, the Magneplane designers concentrated on banking angle, curve layout, power requirements, and performance profiles. Consideration of the vehicle and guideway concepts as briefly summarized in the four alternatives, will reveal that the Magneplane design lends itself to being the easiest geometry for banking.

From an overall basis it can be noted that with respect to vertical geometry, as it applies to guideway design and performance, essentially it is not treated in any of the designs. Also, the authors have gleaned, from comments made in the studies here and there that are unlikely that the guideway will follow any highway right-of-way for any extended distance, primarily because of the tight turns that occur on highways. The high speeds of the MAGLEV system will require large radius turns, with provision for banking (super elevation); this latter topic received little attention in the conceptual design studies, except for Magneplane. As might be expected, Magneplane pointed up its vehicle/guideway system geometry in terms of advantage for banking on turns.

Route factors that would impact construction are being looked at in detail as a part of a separate study under the ongoing U.S. Army CERL assessment program. Indeed, in many cases, in evaluating 'route type' factors, it would be difficult to be more specific until a specific route was chosen, but some general observations can be made, and these follow in this section.

\subsection{Existing Routes}

Certain of the conceptual designs suggested that the routing might be along the median of interstate highways or other corridors, for example railroad rights-of-way. On the other hand, there is a reason to believe that although some of these corridors might be viable under certain circumstances, as noted earlier herein in this section of the summary report, in most cases the curvatures associated with turns on these routes would be totally unacceptable for a high speed vehicle. Most high speed ground transportation systems would be unable to follow existing highway-railway routes other than possibly when it is convenient (or necessary) through congested urban areas.

\subsection{Rivers and Tunnels}

The route studies would need to give special attention to the crossing of rivers, estuaries, and soft ground, in the sense of not only long-term performance (settlement and stability) of the piers (and guideway) under such situations, but also in the sense of safety measures for egress of people in the event of emergencies. Wetlands received special attention in the Magneplane design.

Likewise, tunnels will necessary have to receive special attention in the sense of adequate clearance for vehicles, airflow and acoustic effects, to prevent difficulties with the vehicle systems as they traverse tunnels. In the case of air flow around vehicles in a tunnel, Magneplane, Grumman, and Foster-Miller each made mention of the importance of the problem, especially for high speed vehicles. Guideway support systems in the vicinity of rivers and streams, and factors associated with the use of tunnels, are going to require a great deal more attention as design progresses.

\subsection{Grade Level/Overhead Guideway Plan}

For some designs vehicles tilting will be required on curves. The maximum tilt of the vehicle for the various designs is noted in parentheses followed by the maximum bank angle: Bechtel (15 deg./30 deg.); Grumman (9 deg./ 24 deg.); Foster Miller (12 deg./28 deg.); and Magneplane (no tilt by virtue of the design vehicle-guideway system/ 45 deg.).

\subsection{Horizontal Geometries}

Among the various designs, Bechtel studied the matter of limiting horizontal geometries, and noted further that fiber reinforced plastic (FRP) tendons might be required in the long term in switching systems. Foster-Miller noted that glass reinforced plastic (GRFP) tendons may be needed in the long term on switches. The other designers made particular note of the requirements and singled it out for the future studies.

\subsection{Aesthetics}

As would be expected in a conceptual design, only a limited amount of attention was directed to aesthetics. It became obvious when reviewing the conceptual designs that the geometry of the columns was a big factor in overall aesthetics. For an aboveground facility such as the one contemplated, the matter of aesthetics will be a major concern with regard to the public and governmental entities that are concerned with the location of the facilities and guideways, as well as the overall operation. Probably the two most attractive column systems were the Bechtel canted double-column and cap arrangement, and the Grumman single pedestal. The other two designs called for parallel double columns. Also of importance is the spacing of the columns; in most cases even though some design exercises were undertaken to explore girder depth and span, versus column spacing along the guideway, baseline designs varied greatly. The Bechtel design called for a column spacing of $25 \mathrm{~m}$, whereas the Grumman baseline was $18 \mathrm{~m}$. The Magneplane design appeared to have columns at about a $9 \mathrm{~m}$ spacing, namely quite close, which would seem to pose an aesthetic, if not a cost, concern.

Of equal importance will be attention to the effect that these systems will have on the surrounding land, facilities, housing developments, and industries, in the neighborhood of systems, both from an aesthetic appearance point of view as well as a noise point of view.

In the light of the above-ground power line systems strung across the specific route, much less recent attention to possible electromagnetic effects on people and animals, it 
seems likely MAGLEV will come under intense scrutiny for similar considerations, especially noise and electromagnetic effects on people, both those on vehicles as well as those living and working near such systems.

\subsection{Power Supply}

An aspect of operation and safety that needs attention is the matter of loss of power and measures to handle emergencies loss of power would shut down the system and may require the need for quick egress. What is not so clear from the conceptual designs is whether or not there will be redundant power sources. Propulsion power 'pulsing' were it to occur, could lead to undesirable oscillations of the vehicles during travel; there was modest mention of that possibility in one or more of the MAGLEV designs. Additionally, there is the matter of power cabling; will this cabling be run along or within the guideway, or exterior to the guideway? The security of such a system, much less considerations of magnetic fields associated therewith, will be a consideration. It is realized that these topics are partly operational problems, but the guideway design necessarily will have to include consideration of such matters.

\subsection{Personnel Safety/Emergency Stop}

The matter of safety of personnel (passengers and operating crew) in the event of an emergency stop is one of great concern. Magneplane, Grumman and Foster-Miller give some attention in the conceptual design to walkway concepts, for use in emergencies. Grumman and Magneplane focused attention on a central walkway between the vehicle guideways, although this design raises questions about its viability in the sense of protection for keeping people from falling off the central walkway, including downward egress to exit the system especially in difficult terrain, over water, or in inclement weather. Foster-Miller exhibited a downward (beneath vehicle) exit arrangement. Clearly, safety concepts with regard to passengers are a matter that is going to require a great deal of additional attention.

\subsection{System versus Environment}

Another factor with regard to the route that will need attention is the differing climates through which the system would necessarily operate, perhaps from warm through cold climates. In such cases, the route will need to be evaluated for its ability to accommodate the varying types of weather and environments that it may encounter. For example, Bechtel noted that icing could create problems for its vehicle/guideway design. Magneplane studied and problem, and especially debris related matters. Grumman considered weather as no problem, probably because of the large gap maintained between the guideway and vehicle. In the case of Foster-Miller, the open part of the bottom of the guideway serves to eliminate much of the problem with weather. It is apparent that all designers were quite conscious of the problem that might exist, for they gave great attention to sensors for monitoring such effects.
In some respects the Magneplane designs will preclude some of the difficulties with inclement weather, because of its open truss design, although the details of the 'trough' area as yet not clear. At the other extreme, the Foster-Miller design suggests major problems because of the vertical sidewall and the extreme small clearance between the sidewall and vehicle, measured in centimeters apparently.

\subsection{Environmental}

All of the designers gave consideration to the environment to some degree, some in more detail than others, and especially with regard to the operation of the system. Among the environmental effects requiring attention are such factors as earthquakes (seismic effects), wind (straight winds up through tornadoes), rain, ice, snow, lightning, dirt, debris, and flooding, and their affiliated effects, including such items as slope stability and liquefaction. Detailed design criteria will need to be developed to cover these and other factors.

External vehicle/system factors include effects of noise on passengers and route environs, as well as vibrations/motions of the vehicle as it would affect ride quality need investigation. Similar comments pertain to electromagnetic field effects, as they may possibly affect passenger comport and health.

\subsubsection{Wind}

Certain of the designers, but in varying degrees addressed high winds. It is perceived that for some of the vehicle guideways systems were the vehicle to stop on a curved guideway sector with significant tilt, high wind could lead to a stability problem. This matter was examined only superficially and there is reason to believe from operation of regular railway systems that this can be a major design factor. One could guess it might be critical for the FosterMiller concept with the small clearance between the sidewall and the vehicle, especially on curves, if not straight guideway runs. Bechtel identified wind as a problem to be dealt with.

\subsubsection{Earthquakes}

Earthquakes irrespective of whether or not their source is near or far can pose a major problem, not only in the sense of vibration of the guideway system and therefore the operating vehicle, but also from standpoint of rotational motion of the aboveground supports (pillars), differential ground settlement arising from slope instability or liquefaction, and a host of other related affects.

Also, some attention is given to the matter of detecting earthquakes, and the sensing systems that would be needed on high-speed MAGLEV systems, much in the same way that the Japanese designers developed for their high-speed trains. 


\subsubsection{Debris and Dirt}

From an operational point of view debris and dirt, for example, can have a major effect upon the operational characteristics of the system and these have not been addressed in any great depth in any of the reports that we have reviewed. The debris could originate form environmental effects (i.e. wind) or be placed by people (sabotage). Some type of detection system to counter those effects would seem desirable.

\subsubsection{Noise and Vibration}

Noising arising from operation of these high-speed systems can be a factor, especially in urban areas. It was noted that all four designers (Bechtel, Magneplane, FosterMiller and Grumman) gave some attention to this matter; Grumman paid particular attention to aero-acoustic noise, as well as aerodynamic studies.

\subsubsection{Safety}

Safety is mentioned in all four conceptual designs, but receives the most comprehensive attention in the Bechtel and Grumman submissions. Of great concern, of course, is the matter of derailment as it might occur at switches, and more unlikely (but possible) in the event of ground motions arising from unanticipated settlement, slides, or possibly earthquake effects. The safety of passengers and operating personnel, in case of emergencies, is addressed under the Section 2.1 on Route.

\subsubsection{Electromagnetic Effects}

Electromagnetic effects were singled out by all four design groups as being a major factor, especially as it might affect passengers, much less those staff and maintenance workers located near the vehicles at stations.

A factor that was not noted by the reviewers of the design submissions is electromagnetic effects in the sense that the maglev guideway system is a 'string'. Strings crossing large sectors of the earth can have magnetic effects induced in them to such an extent that this can be a factor of design concern with regard to the performance of the electrical systems themselves, as well as corrosion of metals in the system. Telluric currents may need study in the next phase of the design.

\section{Conceptual Guideway Designs of Four Alternatives}

All four of the major design teams paid varying degrees of attention to the design criteria and preliminary design of the guideways. The assessment that follows begins with a short description of each of the four conceptual guideway designs; it suffices to note that the reviewers are aware that in many cases the descriptions and studies must be general a most in light of the uncertainty of the vehicle design, including levitation and motivation.

\subsection{Bechtel Corporation and Subcontractors}

The Bechtel design is consisted of two parallel eight span continuous prestressed haunched concrete box girders (each supporting a vehicle transit system) shown in Fig.1. The girders, in turn, are supported on a bent with sloped legs, tapered as viewed in elevation, supported in the general case on integral spread footings. The column bents have a spacing of $25 \mathrm{~m}$. The girder connection to the adjacent continuous span would be with a simply supported partial interior span that would permit adjustment during construction and thereafter. Bechtel typical guideway structural details are given in Fig. 2.
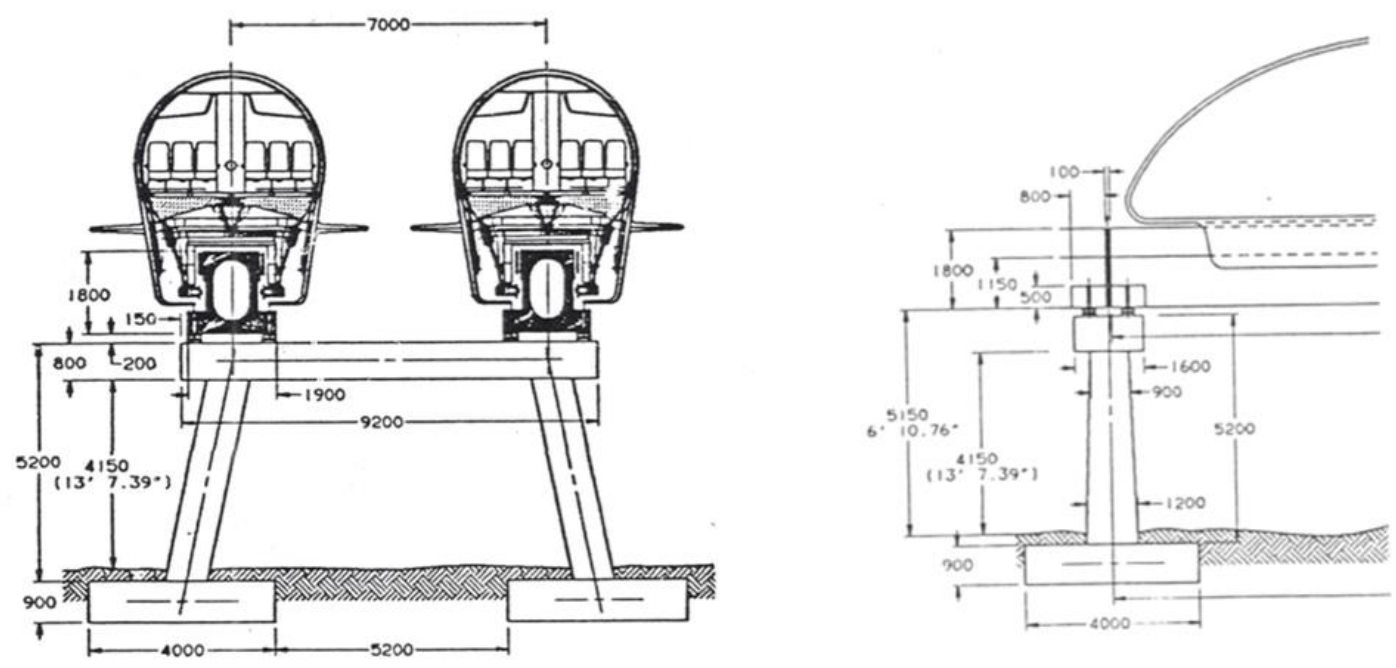

Fig. 1. Bechtel design typical guideway sections. 

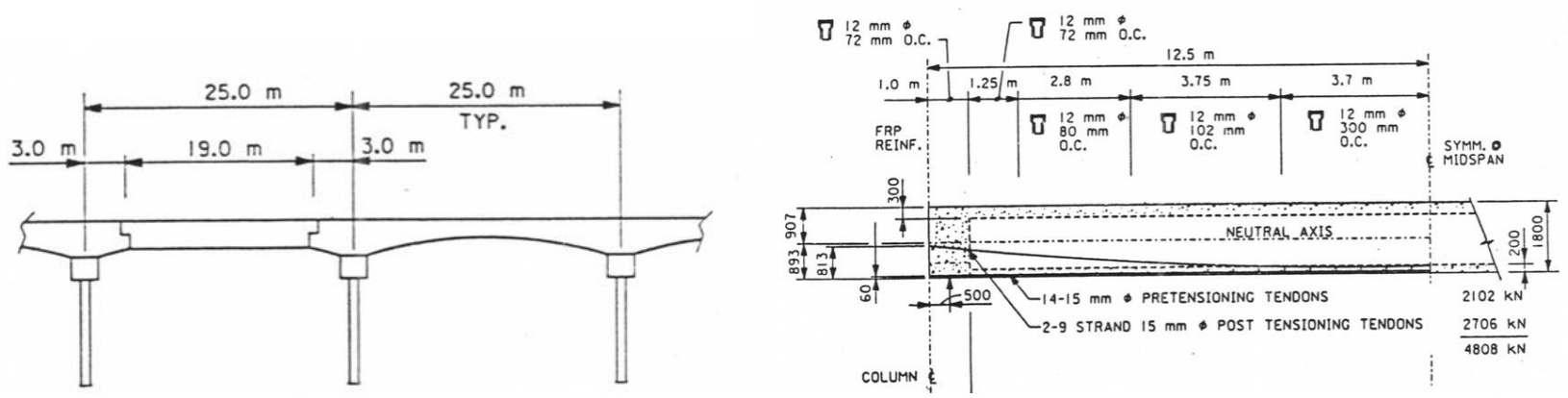

Fig. 2. Bechtel design typical guideway structural details.

The authors note that the bearing surfaces at the top of the bents are large with provision for adjustment, and providing a platform in the event of unexpected deformation, and thereby safety. Moreover, the load path from the beams is directly down through the columns of the bent, providing a stiff, aesthetically pleasing system. These closed box sections have the advantage of being stiff in torsion, and would lead to reduce deflection, the overall effect being a smooth base for the vehicle ride.

The levitation system is based on a 'flux canceling' electrodynamics (EDS) system in which superconducting coils on the vehicle interact with a ladder-like structure on the guideway, with the latter providing suspension and some guidance of the vehicle. No information was found that related to clearances above the rail, or at the sides.

\subsection{Magneplane and Subcontractors}

Several schematics are shown in Fig.3 for the Magneplane guideway system. In general form the longitudinal guideway structure consists of continuous steel truss 'beams' of varying form. The 'two' guideway systems are supported in turn on a concrete cap girder, and thence down to earth on two concrete columns to a spread footing or pile foundation. The guideway trusses were noted to be continuous over two spans. The columns spacing along the guideway was just a little over $9 \mathrm{~m}$, meaning that the columns are spaced relatively close along the guideway. In some configurations the single-track system is shown supported on single concrete columns. Magneplane typical guideway structural details are given in Fig.4.

The 'magway' consists of aluminum box beams supported in turn by the steel truss members. Concrete beams also were considered, but were ruled out for cost reasons. The unusual and novel feature of this vehicle system is that it essentially is supported on a hexagonal base, permitting it to move, as required to handle curves and other system requirements.

The levitation is noted to be of the EMS (attractive) type, although levitation and propulsion were noted as not having been studied in detail as a part of the conceptual study. The clearance during operation is currently planned to be about $0.15 \mathrm{~m}$ or $15 \mathrm{~cm}$, the largest of any of the systems.
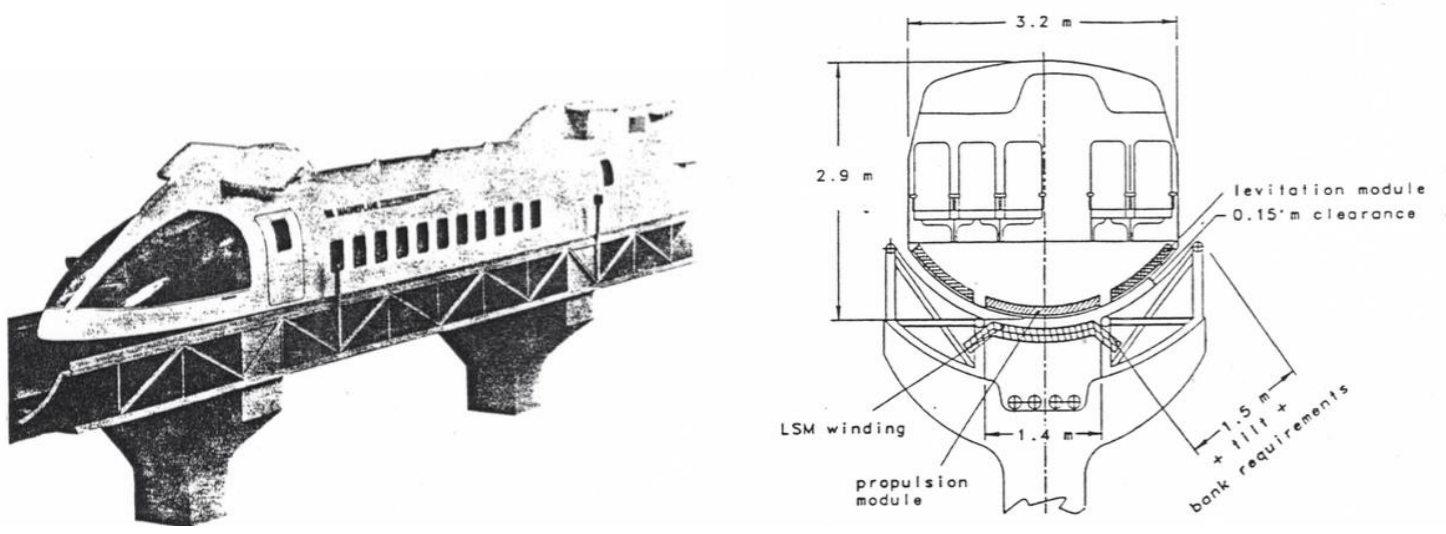

Fig. 3. Magneplane design typical guideway sections. 

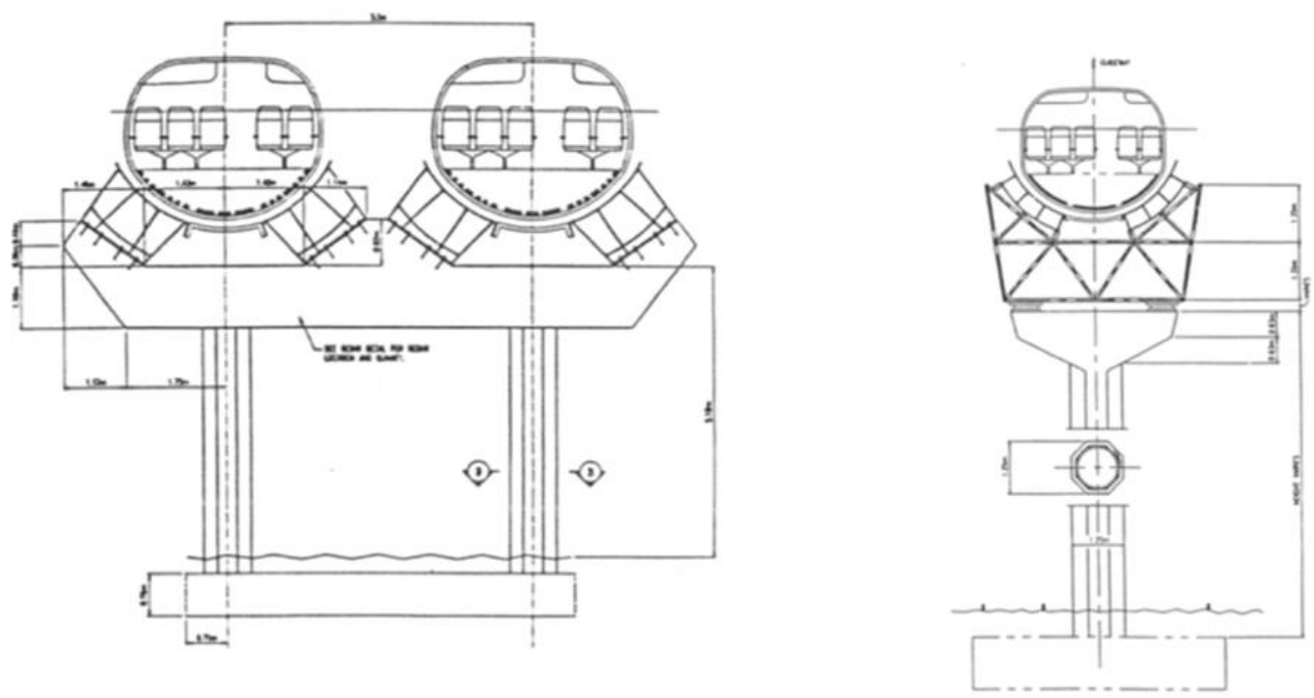

Fig. 4. Magneplane design typical structural design.

\subsection{Grumman and Subcontractors}

The Grumman guideway system as shown in Fig.4 is envisioned as a twin-track guideway, with a central prestressed box girder (the so-called spine girder concept), simply supported on pedestals, and in turn with the maglev rail supported on cantilever members in turn supported by the central prestressed concrete box girder. The depth of the prestressed concrete central box girder varies, and is dependent on the span length. The column is founded on a rectangular concrete footing that is many cases will be on piles; various types of footing supports were examined.
Computations were made for simply supported spans, of varying lengths of $18 \mathrm{~m}$ (baseline) up to $49.5 \mathrm{~m}$. The loadings, for the most part, from the vehicle are unsymmetrical as can be seen visually in the figures. Grumman typical guideway structural details are given in Fig.6.

The vehicle levitation system is of the EMS type, namely an 'attraction system' that helps remove some of the electrical filed from the cabin area. Other advantages claimed are that it leads to a uniform load distribution along the full length of the vehicle, leads to smaller pole pitch, levitation at all speeds, and leads to a wrap around configuration that promotes safety during operation.

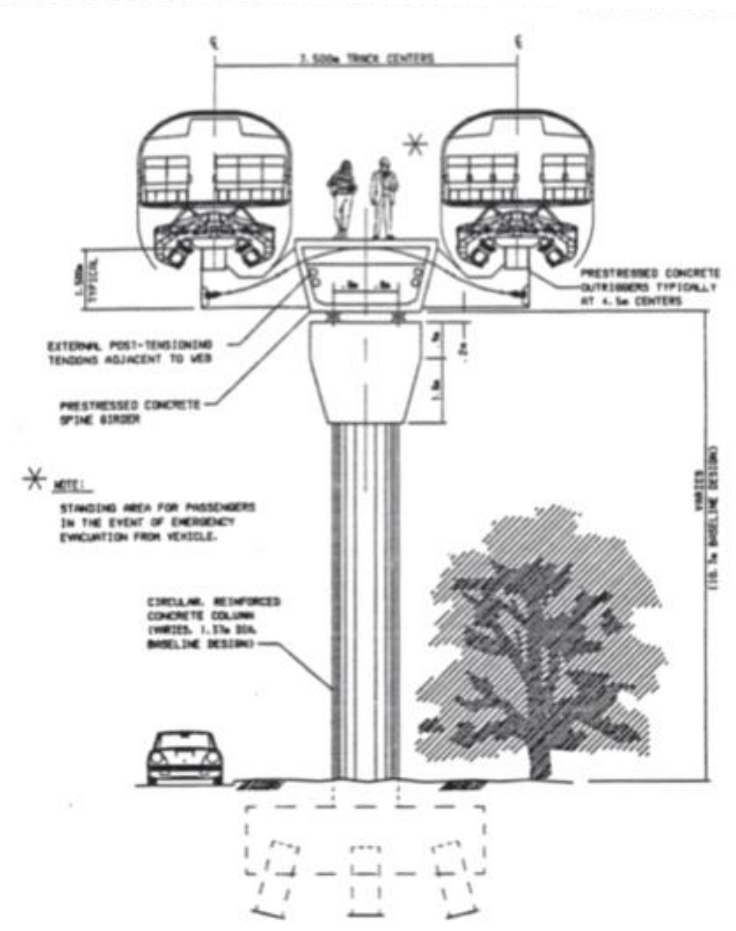

Fig. 5. Grumman design typical guideway sections. 

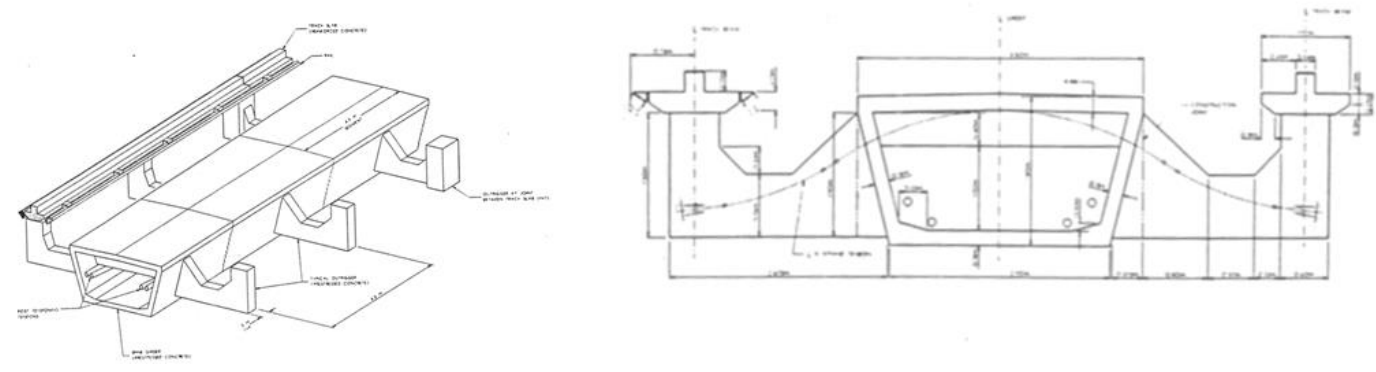

Fig. 6. Grumman design typical guideway structural details.

\subsection{Foster-Miller and Subcontractors}

As gleaned from the description shown in Fig. 7 and design report [5], the preferred guideway system is one of modular construction and has twin hollow beams connected by structural diaphragms. Foster-Miller typical guideway structural details are given in Fig.8. The designers note the following advantages of their system: (1) Open bottom eliminating problems of ice, snow and debris accumulation; (2) wide 'track gauge' for vehicle stability; (3) sidewalls offering significant protection of vehicles under crosswinds and gusts and (4) most convenient for sidewall levitation scheme.

The vehicle system is based on the EDS system (repulsive principle); the conceptual design calls for a nullflux sidewall levitation system. The propulsion is by a locally switched advanced linear motor. The gap clearance is reported in one table to be as great as $7.5 \mathrm{~cm}$. The clearances between the vehicle and the levitation walls are quite small (measured in $\mathrm{cm}$ ), suggesting major problems if there are any distortions in the system, at super elevation/curve geometries,etc.
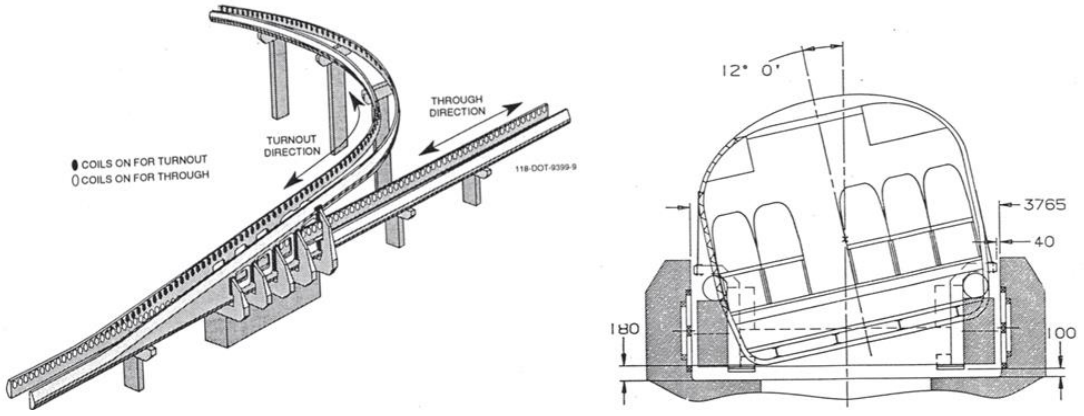

Fig. 7. Foster-Miller design typical guideway sections.
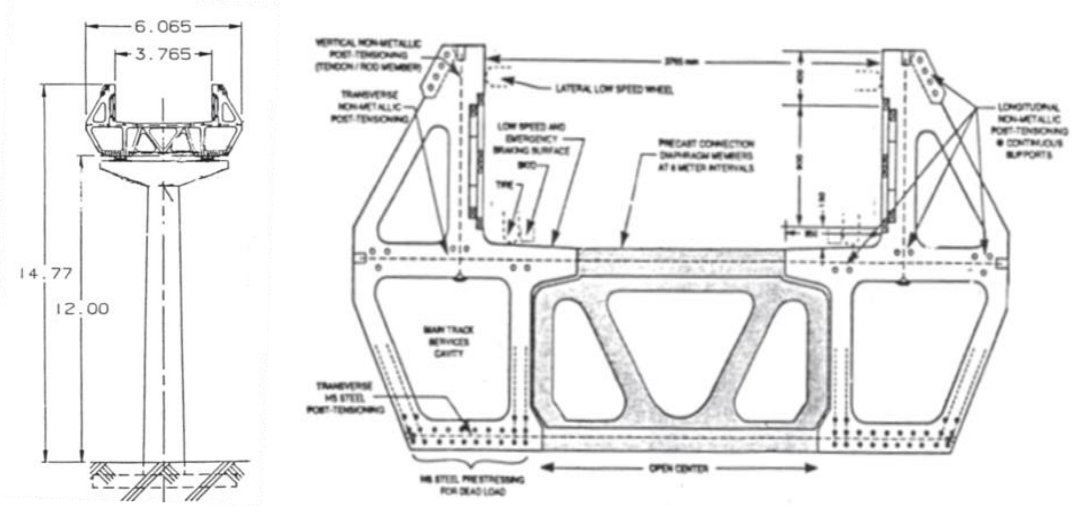

Fig. 8. Foster-Miller design typical guideway structural design.

\section{Guideway Structural Design Concepts for MAGLEV}

\subsection{Loadings}

All of the designers provided some degree of attention to the loading criteria to which the guideway should be designed. Grumman and Bechtel probably provided as much information as anyone. With respect to seismic effects, all mentioned it, but probably Bechtel was most realistic in noting that no transportation system is completely invulnerable from seismic effects. Some indicated code design requirements, which would be the minimum under any circumstances. Bechtel called out a need for sensors to provide warning of seismic activity, and intelligence for being aware of possible alignment problems and disruptions that might be associated with seismic activity. Fatigue 
considerations were not singled out as an important problem at this stage. Other considerations that might be considered 'loadings' in a generic sense are discussed under VehicleGuideway interaction, below.

Vibrations, magnetic forces, and braking forces were considered in some detail by Bechtel as well as FosterMiller. Magneplane had a few comments on vibrations and braking forces, as did Grumman.

\subsection{Deflection, Camber and Tolerances}

In the design of the guideways, attention will be given to the matter of the deflection tolerances and how much camber should be initially built in to take account of vehicle loads and/or creep and thermal affects. Such considerations refer not only vertical effects, in the sense of vertical loads on the guideways, but also to horizontal effects in the sense of straightness of the guideway members, and the control on the guideway system as vehicles traverse bends in the system.

This is an important topic that in the next level of design will need detailed attention both with respect to vehicle/guideway system operation, but particularly to longterm performance and maintenance considerations.

\subsection{Construction of the Guideway}

Particular notice was made of attention paid to construction considerations. In that regard, Bechtel and Grumman clearly addressed the most fully problems in this topical area, and included attention to connections at all levels, and some attention to foundation conditions in this regard. Foster-Miller comments on construction were much less exhaustive in term of treatment and thought, as compared to the other designers. Magneplane comments were minimal at best.

\subsection{Vehicle-Guideway Interaction}

All of the designs, in one way or another, address the matter of vehicle-guideway interaction, which is important from the standpoint of the operation of the vehicle and the comfort of the passengers, as well as system safety and ride comfort. The vehicle-guideway interaction problem increasingly complicated as the speed of the vehicle increases, both from the operational/aerodynamic aspect, especially as they relate to the operation of the system. All of the designers gave some degree of attention to guideway imperfections, such as those that might arise from changes of alignment between spans, or 'bumps' associates with debris. Thermal effects were noted to be important, especially in the Magneplane presentation where high thermal gradients were noted in connection with vehicle operation; others had lesser attention, with Grumman, so far as the reviewers could tell, giving little attention to this topic apparently.

External factors that could affect the performance, in addition to debris that might be deposited by humans, would include ice, snow, debris deposited by wind, misalignment arising from seismic activity, and thermal effects.

\subsection{Foundation and Pillars}

Various designs gave different attention to the foundations for the pillars or columns that support the guideway system. Probably the magneplane group did the most study in this regard, but all of the studies need additional attention to the different forms of foundations that would be required for an elevated system of this type. It was noticed that the conceptual designs thus far have not given much attention to matters of multiple support of the foundation pads (an exception is Magneplane that showed some multiple piles) to preclude long term settlement and/or rotations that might occur under environmental effects, much less settlement and other factors associated with the guideway operation. It can be envisioned that the footings, a large part of the construction cost, can range from slab on grade in some cases, to those supported on drilled caissons, or on piling. The foundations will need to be firm to resist environmental effects arising from earthquakes, wind, flood, etc. Hopefully in view of the large number of such installations, the cost can be reduced to a degree through novel prefabrication techniques. It is our belief, based on our study that the foundation systems for this elevated guideway system will turn out to be somewhat more complicated, as well as more expensive than has been indicated thus far in the studies.

\subsection{Guideway and Pedestals}

The design of the pedestals or columns varied between the various conceptual designs by reason of the fact that they supported different types of guideway/beam systems. The Bechtel design, as described earlier, uses a canted pair columns that form a frame; the Grumman design employs a single pedestal. The Magneplane and Foster-Miller designs appear to center on double vertical columns, with the caps independent or tied as the case may be. As a result, little can be said about the pedestal designs except to emphasize that these column guideway systems are inverted pendulums in the sense of dynamic effects arising from earthquakes and, thus, will require great attention, not only in terms of the stability of the structural systems, but also their actual design to insure that they can accommodate the lateral forces that arise from vehicle, wind, earthquakes, as well as other unequal and unsymmetrical loadings. In this sense, the Bechtel design is the more stable of those proposed.

In the case of the girders, these ranged in the conceptual design from simple spans (i.e. Grumman) where considerable attention was given to end effects, rotations, creep, settlement, differential settlement, etc., although it was not clear whether or not Grumman had considered twist around the rail, to box girders in some cases, and conceptually continuous girders (i.e. Bechtel). There is a great deal of design and construction trade off that needs to be considered in this area in the sense of construction such systems, as well as handling adjustments with time. Obviously, in the case of simple beam systems, there can be difficulties, as has been experienced with some monorail systems in the United States at the discontinuities that occur at the ends of the girders, as well as the effects of creep and thermal effects (especially in 
areas of the country where there is great temperature extremes). Novel designs were put forth, for example, Magneplane employed a hexagonal guideway support system for their vehicles, which included a truss, a linear arrangement that in essence lightens the guideway system, but perhaps leads to other problems. As an example, it was not so clear in the case of the Magneplane design what may happen in the case of ice, snow and rain in the sense of the vehicles; in one sense, it seemed to be attractive from that standpoint, and in another sense it was not so clear that these factors could be handled as easily as suggested. Another important aspect of the design of the girders is that of the continuity and in a sense continuous spans going across a half dozen or so girders would make it possible to have more control over the reliability and ride comport. On the other hand, the adjustments needed for effects of creep, shrinkage and thermal effects might be more difficult to handle, although this was explored in some degree with prestressing in the case of Bechtel and Grumman. Thus, we see a range of considerations in the possible construction of the guideways.

Another aspect of the design that has needed attention, of course, is that some of the designers have given attention to fiber reinforced plastic (FRP) or glass fiber reinforced plastics (GFRP) as being a reinforcing material to cut down on electromagnetic interference and effects with regard to levitation and propulsion. This is a subject needing additional attention for the studies thus far on FRP have shown good, as well as not-so-good, characteristics with regard to creep, long-term strength, and perhaps thermal behavior.

In terms of general treatment, reinforcing, restressing and anchorage all received some degree of attention, in the most detail by Bechtel, and in lesser detail by Foster-Miller. No significant inclusions were noted by Magneplane and Grumman. This topical area will require great attention in the next design phase, not only in the sense of initial construction, but also in the sense of maintenance adjustments to the system over time to insure adequate system performance. Guideway design requires great additional study in light of the construction costs involved. It may well prove advisable to build short trial sections for pilot use/testing in widely different climates.

\subsection{Thermal Effects}

Thermal effects need a great deal of additional attention. None of the conceptual designs really addressed that question in any great detail. To their credit Magneplane did describe some schemes for use of aluminum materials to dissipate heat they envisioned being generated with their system. Such effects are not only important in the case of pedestals and girders, especially in the sense of their structural adequacy, but also it has an effect upon the vehicles, vehicle guideway interaction, and possibly guideway expansion problems.

\subsection{Air Gap}

The vehicle systems investigated thus far call air gaps ranging from as large as a $15 \mathrm{~cm}$ levitation gap in the case of Magneplane (but $5 \mathrm{~cm}$ was more probable the report indicated) to $4 \mathrm{~cm}$ in the case of Grumman. In any case, whatever the system, whether it be a repulsive or attractive electromagnetic levitation system, the matter of clearances is going to be one of great concern in connection with the design and maintenance of the guideways, and especially operation of the systems. This gap hopefully will provide for differences in deflections and alignments of the guideways, at joints in the guideway, as well as accommodation of debris, ice and snow. Laterally in the case of Foster-Miller, the vehicle appears to have little clearance with the guideway, especially if the vehicle tilts on super elevated curves, or in the wind. Thus, there is a wide range of parameters that must be considered in terms of support of the system.

\subsection{System Stability and Rocking Analysis}

Attention was given in some of the reports, especially Bechtel, with regard to the vehicle instability that might occur under normal operation or in taking curves. Wind effects also received consideration, especially in the Bechtel design with respect to rocking considerations. That is one kind of analysis that needs more study. At the other extreme, system stability in general, for example the keel effects need to be studied; Magneplane did, for obvious reasons, look into this matter, but the findings would appear questionable. In the Magneplane design, one can envision a rolling/swooping effect as the vehicle traverses the guideway, which could be of concern not only to operation but also to rideability. Also, these stability effects are important with regard to the operational reliability, especially in terms of the gap that must be maintained for satisfactory operation.

\subsection{Emergency Stops}

Certain of the designs did pay attention to the guideways with regard to the emergency stops. Bechtel concentrated on preferred stop points, or safe stop points, where the vehicles could stop and efficiently handle the passengers.

\subsection{Switching}

Most of the designs considered switching arrangements for their particular vehicle design, but, at this point in time, it is difficult to envision how these would be handled with the guideways that were proposed and (or the terminals that were envisioned. Since some of the switching will occur at high speed and some at low speed, varying criteria will come into play. This is a very difficult technical aspect of design.

\subsection{Stations and Facilities}

All conceptual designers mentioned stations and facilities, although Grumman is the only one to spend some limited space on the matter. It is appreciated that these are more 'routine' in the nature, and would be addressed. The reviewers did notice some attention to passengers and public safety, in term of addressing the 'standoff distance' at stations, from the standpoint of avoiding electromagnetic effects. 


\section{Specific Observations on Guideways}

\subsection{Magnetic Effects}

Among the effects needing more study in the future were magnetic effects (on passengers and operating personnel), vibrations, air gaps, construction tolerances, keel effects, and suspension (especially for emergency conditions). Every report indicated further studies of this topic are needed. Each of the four conceptual designs studied the technical details associated with magnetic effects, on passengers as well as crew and others in the immediate vicinity. Quite different details are noted for each of the designs, as would be expected, each pointing up the advantages of this system.

\subsection{Vibration}

All four designs looked extensively into vibrations as they affect ride quality and the frequencies of such vibrations. The frequencies will be important factors in assessing the vehicle/guideway interaction, vehicle performance, passenger comfort, and long-term maintenance.

It appeared that although some attention to wind had been given in the conceptual designs, in some instances for the guideway curvatures and tilts envisioned, the vehicles were clearly unstable. Bechtel took pains to address this point.

\subsection{Air Gap}

This topic was addressed in the earlier Section 4.8 .

\subsection{Keel Effect}

The keel effect, as it affects the stability of the vehicle, is an important factor. The matter is of particular importance to those systems using large clearances, as for Magneplane. Somehow, in the design process, there will need to be some balance between the air gap associated with levitation and stability of the vehicle under all of its operating modes. Considerations will not only focus on a gap to preclude difficulties with debris, ice and other factors already identified, but also the important matter of safety of control of the system at high speed to preclude accidents of various types, including 'running off the guideways' especially for some designs.

\section{Maintenance/Repair}

Although there was passing mention in the conceptual design reports given to maintenance and repair, little information of that type were presented. Perhaps the most attention to service vehicles and surveillance is that given in the Bechtel [2] and Grumman [4] reports. This is a major topic needing detailed consideration, not only with regard to the vehicles and guideways, but the power system and its reliability, and the viability of the systems under the environmental effects outlined previously. The Bechtel report [2] did give some attention for example, to a service vehicle concept, which would be needed for the safe and efficient operation of a MAGLEV System.

Numerous questions arise. For example, will corrosion of the guideway electronics be a factor? Will surface deterioration of concrete, as well as reinforcing corrosion be a problem? How will adjustments in alignment be made?

\section{Conclusion}

High-speed trains constructed on guideway structures for ground transportation system as magnetic-levitation system require the important conceptual structural design parameters. There are variety of design parameters not only design loads, selection of structural section, but also disaster management, engineering planning and design for unexpected loads as well as maintance and repair of the system. Specific observations on guideways are discussed as loads including seismic loads, vibrations, magnetic forces, breaking forces as well as considering imperfections due to alignment or bumps in case of debris, ice, snow and rain; operational/aerodynamic effects considering vehicleguideway interaction; deflection, camber and tolerances both in vertical direction and in the horizontal direction, vehicle loads and/or creep, fatique, and thermal effects must be considered. On the other hand, construction of guideways, route type factor for a specific route including special passes such as tunnels, lakes; foundation systems, problems in tropical areas, structural connections will be considered. To control the system and uncertainties, sensors to provide warning of seismic activity, intelligence for being aware of possible alignment problems, GIS systems are necessary. Vehicle-guideway interaction is another important parameter for the operation of the vehicle, comfort of the passengers, safety and ride comfort. In the design of girders continuity is also important to redistribute the additional unexpected forces and displacements. Other design parameters are air gap, system stability and rocking analysis, emergency stops, switching stations and facilities.

For future studies corrosion of the guideway electronics, deterioration of concrete, corrosion of reinforcement, terror attacks, seismic faults effects, unexpected support settlement, interruption of electromagnetic effects, problems in monitoring and other safety problems will be considered as conceptual design parameters.

\section{Acknowledgements}

This work [1] was undertaken in part under USACERL with University of Illinois, with Dr. Can Balkaya and W.J. Hall. We gratefully acknowledge the assistance provided by USACERL and its staff, and particularly the guidance and oversight provided by Mr. Don Plotkin, USACERL.

\section{References}

[1] W.J. Hall and Can Balkaya, 'Final Report on MAGLEV Guideway Design Review, Task-2 Long Term Performance and Seismic Assessment of Maglev Guideways' USACERL, 1993. 
[2] Bechtel Corporation et al, 'Maglev System Concept Definitions' Final Report to US Department of Transportation, Federal Railroad Administration, 1992. (Co-investigators: Hughes; GM Electro-Motive; Draper Laboratory; Massachusetts Institute of Technology).

[3] Magneplane International Inc., 'System Concept Definition Report for the National Maglev Initiative', 1992. (Co-investigators: Massachusetts Institute of Technology; United Engineers and Constructors; Raytheon Equipment Division; Failure Analysis Associates; Bromwell \& Carrier; Beech Aircraft Corporation; Process Systems International).
[4] Grumman Aerospace Corporation, 'System Concept Definition of a Superconducting Maglev Electromagnetic System' Final Report, 1992. (Co-investigators: Parsons Brinckerhoff; Intermagnetic General Corporation; PSM Technologies; Honeywell; Battelle; Gibbs and Hill; NYSIS.

[5] Foster-Miller Inc., 'Maglev System Concept Definition Report' Final Report, 1992.

[6] Don Plotkin and Simon Kim, 'Maglev Guideway Design Issues' Journal of Transportation Engineering, May/June 1997, 189-191. 